\title{
Mean Comparison Theorem cannot be extended to Poisson case
}

\author{
Jan Večeř \\ Columbia University, Department of Statistics, New York, NY 10027, USA \\ Email: vecer@stat.columbia.edu \\ Mingxin Xu \\ Carnegie Mellon University, Department of Mathematical Sciences, Pittsburgh, PA 15213, USA \\ Email: mxu@andrew.cmu.edu
}

This version: March 28, 2004

\begin{abstract}
In this paper, we show that the Mean Comparison Theorem which is valid for Brownian motion, cannot be extended to Poisson process. A counter example in the Poisson case, for which the Mean Comparison Theorem does not hold, is provided.
\end{abstract}

\section{Introduction}

Problems of comparing expected values of convex functions corresponding to processes which are related to each other have many applications in stochastic optimal control and in option pricing. For the case of Ito's processes driven by standard Brownian motion, it is true that the process with the largest $d W_{t}$ term in absolute value has the largest expectation if evaluated in any convex function. More specifically, the following theorem of Hajek (1985) holds:

Theorem 1 (Hajek's Mean Comparison Theorem) Let $X_{t}$ be a continuous martingale with representation $X_{t}=X_{0}+\int_{0}^{t} \sigma_{s} d W_{s}$. Assume that for some function $\rho$ on $\mathbb{R}$, we have $\left|\sigma_{s}\right| \leq \rho\left(X_{s}\right)$ and there exists a unique (in the sense of probability law) solution $Y_{t}$ to the stochastic differential equation

$$
Y_{t}=X_{0}+\int_{0}^{t} \rho\left(Y_{s}\right) d W_{s}
$$

Then for any convex function $\Phi$ and any $t \geq 0$,

$$
\mathbb{E} \Phi\left(X_{t}\right) \leq \mathbb{E} \Phi\left(Y_{t}\right) .
$$

The proof of this theorem is based on representing processes $X_{t}$ and $Y_{t}$ as time changed Brownian motions, see Hajek (1985) for details.

Similar problems arise in comparing option prices, which are computed as expectations of convex payoffs corresponding to the stock price process. It has been shown in the Brownian motion setup that under quite general conditions, stocks with higher volatility (which corresponds to the leading $d W_{t}$ term) have higher price and thus higher expected convex payoffs. This case has been 
studied for instance in El Karoui, Jeanblanc-Picque and Shreve (1998), or in Hobson (1998).

Recently there has been some effort to extend these results to models which include jumps, Poisson process being the simplest example. Interestingly enough, the comparison results were mostly affirmative for problems considered. Kijima (2002) proved that if the jump size is independent to the driving Poisson process and the expectation of the jump size is fixed, option prices are increasing as a function of convex expectation of the jump size for any given convex function. Several results involving comparison of option prices under jump-diffusion models were obtained by Henderson and Hobson (2003). Their goal was to compare prices of options computed under different choice of risk neutral measures. They also showed that option prices are not monotonically increasing in the jump intensity if there is more than one possible jump size. Večer (2000) showed that the Mean comparison theorem holds for Passport options if the stock is driven by a Poisson process and when the contract is terminated at the time of $k$-th jump.

The present paper shows that Hajek's Mean comparison theorem cannot be extended to the Poisson case in general. Although there are some positive results in this direction under various conditions mentioned in the above listed literature, we found a counter example for a specific choice of random processes and for exponential payoff function. In the following text, we assume the driving process is Poisson with fixed intensity $\lambda$ and we show that the dominance of jump size does not imply dominance of the expected convex payoff.

\section{Counterexample in the Poisson model}

\section{Theorem 2 (Negative Result of Mean Comparison Theorem for Poisson Process)}

There is a convex function $\Phi$ and processes $X_{t}$ and $Y_{t}$ with representation

$$
X_{t}=X_{0}+\int_{0}^{t} \sigma_{s} d\left(N_{s}-\lambda s\right)
$$

$$
Y_{t}=X_{0}+\int_{0}^{t} \rho\left(Y_{s-}\right) d\left(N_{s}-\lambda s\right)
$$

where $\rho$ is a function on $\mathbb{R}$ with $\left|\sigma_{s}\right| \leq \rho\left(X_{s-}\right)$, so that

$$
\mathbb{E} \Phi\left(X_{T}\right)>\mathbb{E} \Phi\left(Y_{T}\right)
$$

for some $T>0$. In particular, for a given fixed time $T>0$, the following functions represent one possible choice. For constants $\epsilon>0, \alpha_{0}>\frac{1}{2} \epsilon \lambda T$, and c large enough, let

$$
\begin{gathered}
\sigma_{t}=\alpha_{0} 1_{\left\{0 \leq t \leq \frac{T}{2}\right\}}+c 1_{\left\{X_{t-} \leq-\alpha_{0} \lambda \frac{T}{2}-c \lambda\left(t-\frac{T}{2}\right)\right\}} 1_{\left\{\frac{T}{2}<t \leq T\right\}}, \\
\rho\left(Y_{t-}\right)=\left(\alpha_{0}+\epsilon\right) 1_{\left\{0 \leq t \leq \frac{T}{2}\right\}}+c 1_{\left\{Y_{t-} \leq-\alpha_{0} \lambda \frac{T}{2}-c \lambda\left(t-\frac{T}{2}\right)\right\}} 1_{\left\{\frac{T}{2}<t \leq T\right\}},
\end{gathered}
$$

and $\Phi(x)=e^{x}$.

Remark 3 The proof of this result is based on the following idea. On the set $\left\{N_{T / 2}=0\right\}$, we have $X_{T / 2}>Y_{T / 2}$. We can amplify this effect at time $T$ by choosing a large $c$ in (2.6) and (2.7). For a particular choice of convex function $\Phi(x)=e^{x}$, it is possible to make this effect dominant. 
Proof of the Theorem. We have clearly $\left|\sigma_{t}\right| \leq \rho\left(X_{t-}\right)$ for the above choice of parameters. We can also obtain representation for both processes $X_{T}$ and $Y_{T}$ in terms of the underlying Poisson process. Let us denote $M_{t}=N_{t}-\lambda t$, and let $\tau$ be the stopping time of the first jump of the Poisson process. We have

$$
\begin{aligned}
& d X_{t}= \alpha_{0} 1_{\left\{0 \leq t \leq \frac{T}{2}\right\}} d M_{t}+\alpha_{1}(t) 1_{\left\{\frac{T}{2}<t \leq T\right\}} d M_{t}, \\
& \text { where } \quad \alpha_{1}(t)=c 1_{\left\{X_{t-} \leq-\alpha_{0} \lambda \frac{T}{2}-c \lambda\left(t-\frac{T}{2}\right)\right\}}=c 1_{\left\{X_{t-}=-\alpha_{0} \lambda \frac{T}{2}-c \lambda\left(t-\frac{T}{2}\right)\right\}}, \\
& \text { i.e. } \quad \alpha_{1}(t)=c \Leftrightarrow N_{t-}=0 ; \\
& X_{T}= \alpha_{0}\left(N_{\frac{T}{2}}-\lambda \frac{T}{2}\right)-c \lambda \frac{T}{2} 1_{\left\{N_{T}=0\right\}}+c 1_{\left\{N_{T / 2}=0\right\}} 1_{\left\{N_{T}>1\right\}}\left(1-\lambda\left(\tau-\frac{T}{2}\right)\right) ; \\
& d Y_{t}=\left(\alpha_{0}+\epsilon\right) 1_{\left\{0 \leq t \leq \frac{T}{2}\right\}} d M_{t}+\beta_{1}(t) 1_{\left\{\frac{T}{2}<t \leq T\right\}} d M_{t}, \\
& \text { where } \quad \beta_{1}(t)=c 1_{\left\{Y_{t-} \leq-\alpha_{0} \lambda \frac{T}{2}-c \lambda\left(t-\frac{T}{2}\right)\right\}}, \\
& \text { i.e. } \quad \beta_{1}(t)=c \Leftrightarrow N_{t-}=0, \quad \text { when } \quad \alpha_{0}>\frac{1}{2} \epsilon \lambda T ; \\
& Y_{T}=\left(\alpha_{0}+\epsilon\right)\left(N_{\frac{T}{2}}-\lambda \frac{T}{2}\right)-c \lambda \frac{T}{2} 1_{\left\{N_{T}=0\right\}}+c 1_{\left\{N_{T / 2}=0\right\}} 1_{\left\{N_{T}>1\right\}}\left(1-\lambda\left(\tau-\frac{T}{2}\right)\right) .
\end{aligned}
$$

Take

$$
\Phi(x)=e^{x} .
$$

Since for $0 \leq t \leq \frac{T}{2}$,

$$
\begin{aligned}
P\left(\tau \leq t \mid N_{T / 2}>0\right) & =P\left(N_{t}>0 \mid N_{T / 2}>0\right)=\frac{P\left(N_{t}>0, N_{T / 2}>0\right)}{P\left(N_{T / 2}>0\right)} \\
& =\frac{P\left(N_{t}>0\right)}{P\left(N_{T / 2}>0\right)}=\frac{1-e^{-\lambda t}}{1-e^{-\lambda \frac{T}{2}}}
\end{aligned}
$$

we can compute

$$
\begin{aligned}
E\left[\Phi\left(X_{T}\right)\right]= & E\left[e^{\alpha_{0}\left(N_{T / 2}-\lambda T / 2\right)-c \lambda(T / 2) 1_{\left\{N_{T}=0\right\}}+c 1_{\left\{N_{T / 2}=0\right\}} 1_{\left\{N_{T}>1\right\}}(1-\lambda(\tau-T / 2))}\right] \\
= & E\left[e^{\alpha_{0}\left(N_{T / 2}-\lambda T / 2\right)} E\left[e^{-c \lambda(T / 2) 1_{\left\{N_{T}=0\right\}}+c 1_{\left\{N_{T / 2}=0\right\}} 1_{\left\{N_{T}>1\right\}}(1-\lambda(\tau-T / 2))} \mid \mathcal{F}_{T / 2}\right]\right] \\
= & E\left[e^{\alpha_{0}\left(N_{T / 2}-\lambda T / 2\right)} 1_{\left\{N_{T / 2}=0\right\}} E\left[e^{-c \lambda(T / 2) 1_{\left\{N_{T}-N_{T / 2}=0\right\}}+c 1_{\left\{N_{T}>1\right\}}(1-\lambda(\tau-T / 2))} \mid \mathcal{F}_{T / 2}\right]\right. \\
& \left.+e^{\alpha_{0}\left(N_{T / 2}-\lambda T / 2\right)} 1_{\left\{N_{T / 2}>0\right\}}\right] \\
= & E\left[e^{\alpha_{0}\left(N_{T / 2}-\lambda T / 2\right)} 1_{\left\{N_{T / 2}=0\right\}}\left(e^{-c \lambda T / 2} e^{-\lambda T / 2}+\left(1-e^{-\frac{\lambda T}{2}}\right) \int_{0}^{\frac{T}{2}} \frac{e^{c(1-\lambda t)} \cdot \lambda e^{-\lambda t}}{1-e^{-\lambda T / 2}} d t\right)\right] \\
& +E\left[e^{\alpha_{0}\left(N_{T / 2}-\lambda T / 2\right)}\right]-E\left[e^{\alpha_{0}\left(N_{T / 2}-\lambda T / 2\right)} 1_{\left\{N_{T / 2}=0\right\}}\right] \\
= & E\left[e^{\alpha_{0}\left(N_{T / 2}-\lambda T / 2\right)} 1_{\left\{N_{T / 2}=0\right\}}\left(e^{-(c+1) \lambda T / 2}+\frac{e^{c}}{c+1}\left(1-e^{-(c+1) \lambda T / 2}\right)\right)\right] \\
& +E\left[e^{\alpha_{0}\left(N_{T / 2}-\lambda T / 2\right)}\right]-E\left[e^{\alpha_{0}\left(N_{T / 2}-\lambda T / 2\right)} 1_{\left\{N_{T / 2}=0\right\}}\right] \\
= & e^{-\left(\alpha_{0}+1\right) \lambda T / 2}\left(\frac{e^{c}}{c+1}-1\right)\left(1-e^{-(c+1) \lambda T / 2}\right)+e^{\lambda(T / 2)\left(e^{\alpha_{0}}-\alpha_{0}-1\right)} .
\end{aligned}
$$

For large choice of $c$, the above expectation will become decreasing as a function of $\alpha_{0}$. Therefore we will have $\mathbb{E} \Phi\left(X_{T}\right)>\mathbb{E} \Phi\left(Y_{T}\right)$. 


\section{References}

[1] El Karoui, N., M. Jeanblanc-Picque, S. Shreve, (1998): "Robustness of the Black and Scholes formula", Mathematical Finance, 8, 93-126.

[2] HAJEK, B., (1985): "Mean stochastic comparison of diffusions", Z. Wahrscheinlichkeitstheorie verw. Gebiete, 68, 315-329.

[3] Henderson, V., D. Hobson, (2003): "Coupling and Option Price Comparisons in a Jump Diffusion model", Stochastics and Stochastics Reports, 75, 3, 79-101.

[4] Hobson, D., (1998): "Volatility mis-specification, option pricing and super-replication via coupling", Annals of Applied Probability, 8, 193-205.

[5] KiJima, M., (2002): "Monotonicity and convexity of option prices revisited", Mathematical Finance, 12, 411-425.

[6] VeČĚr, J., (2000): "Options on a traded account", PhD thesis, Carnegie Mellon University. 